\title{
RENORMALIZATION AND COMPUTATION II: TIME CUT-OFF AND THE HALTING PROBLEM
}

\author{
Yuri I. Manin \\ Max-Planck-Institut für Mathematik, Bonn, Germany, \\ and Northwestern University, Evanston, USA
}

\begin{abstract}
This is the second installment to the project initiated in [Ma3]. In the first Part, I argued that both philosophy and technique of the perturbative renormalization in quantum field theory could be meaningfully transplanted to the theory of computation, and sketched several contexts supporting this view.

In this second part, I address some of the issues raised in [Ma3] and provide their development in three contexts: a categorification of the algorithmic computations; time cut-off and Anytime Algorithms; and finally, a Hopf algebra renormalization of the Halting Problem.
\end{abstract}

\section{Contents}

0. Introduction

1. Enriched programming methods with typing and parallelism: a categorical approach

2. Cut-off regularization and Anytime Algorithms

3. Regularization and renormalization of the Halting Problem

\section{Introduction}

0.1. Regularization and Anytime Algorithms. It is well known that classical theory of computability includes as its organic part phenomena of noncomputability. Namely, an attempt to compute the value of a partially recursive function at a point where it is not defined, might stall the computation forever, but we will never know whether this is so or simply we did not wait long enough ("the Halting Problem is undecidable").

Applied theory of computation deals with algorithms processing finite amount of data into finite outputs. Nevertheless, even in such theoretically safe situations time/memory requirements may make the implementation of a sound algorithm unfeasible.

The celebrated theory of polynomial time computations and discovery of the $P / N P$-problem served as a neutral zone meeting point between theoretical possibility and practical feasibility, and revealed beautiful new mathematical structures. 
However, applied computer scientists consider other possible ways of turning unfeasible computations into feasible ones, known under the code word "Anytime Algorithms". Basically, an "Anytime Algorithm" allows the computation to stop at a feasible time, and supplies the result of such a mutilated procedure with a measure of its quality. See [GrZi] and a nice short introduction [Gr].

In the Sec. 2 of this article, the second installment to the project initiated in [Ma3], it is suggested that theoretically "Anytime Algorithms" can be treated as one of the versions of regularization schemes in Quantum Field Theory: time cutoff (for more detailed description of the whole project, see Introduction to [Ma3]). More precisely, I analyze from this viewpoint results of the stimulating paper by Ch. Calude, M. Stay "Most programs stop quickly or never halt" ([CalSt1]).

One of the themes, that the analogies with renormalization and experience with Anytime Algorithms bring to the foreground in the computation theory, is the stress on the structure of programs determined by the operation "composition of programs" and by the explicit parallelism, that played a key role in our treatment of perturbative renormalization as a model for regularizing computations in [Ma3].

Notice that many standard descriptions of programming methods are not stable with respect to the composition and have no natural means for expressing parallelism.

For example, composition $T_{2} \circ T_{1}$ of two Turing machines, informally defined as computation in which the output of $T_{1}$ becomes the oracular input ("program") for $T_{2}$, is not directly described as a new Turing machine $T_{3}$.

Language-like constructions such as lambda-calculus, being inherently linear/sequential, are not well-suited for expressing options of parallelism.

"Flowcharts" imagery for which I made some propaganda in [Ma3] serves these goals much better. In the Section 1 of this article, I show that the same ideas admit a succinct categorical expression, and suggest that flowcharts constructions from [Ma3] can be interpreted as a constructive existence theorem, to produce what I call "an enriched programming method with unrestricted parallelism" (cf. Definition 1.8.1). This seems to be very much in the spirit of [BaSt].

Finally, Section 3, using some ideas from quantum computation, provides a Hopf renormalization scheme for the Halting Problem.

0.2. Computability as a mathematical structure and its interaction with other mathematical structures. Most of the constructions considered in this paper refer to (un)feasible algorithms with infinite domains/ranges. Devising 
their natural quantitative characteristics and regularization schemes for them, one should keep in mind that they can be roughly subdivided into two large blocks.

$B L O C K A$. This block consists of the inherent problems referring to an infinite constructive world $X$, which depend only on the class of "admissible" recursively equivalent numberings of $X$, and which are the same for all infinite $X$. From the computational viewpoint, any such $X$ can be identified with $\mathbf{N}$ (natural numbers) or $\mathbf{Z}^{+}$(nonzero natural numbers).

A typical example of such an $X$ is some set of finite Bourbaki structures, such words in a finite alphabet, or finite groups, or graphs, or their descriptions, etc. In this context, one uses the Bourbaki description primarily in order to define the class of admissible bijections (numberings) $\mathbf{Z}^{+} \rightarrow X$ in question: they must be informally computable together with their inversions. One aspect of Church's thesis consists in the statement that recursive functions will provide an adequate notion of algorithmic processing of elements of $X$ whenever we can imagine an informal algorithm producing the numbering.

Once it is decided that the role of the respective Bourbaki structure is over as soon as the class of numberings is determined, one can make explicit various secondary structures on $X$ that can be defined exclusively in terms of admissible numberings.

One such structure is the algebra of enumerable subsets of $X$ : definition domains $D(f)$ of partial recursive functions. This family is stable wrt finite intersections and enumerable unions. If one consider these sets modulo finite ones, one can prove interesting results about simple sets, maximal sets etc. For example, maximal $D(f)$ display a striking similarity to the holomorphy domains $V$ in the theory of complex analytic functions of $\geq 2$ variables: in both cases, there are functions defined on $D(f)$, resp. $V$, that cannot be extended to a larger domain.

The proviso "modulo finite subsets" can be very naturally formalized by changing the Constructive Universe $\mathcal{C}$ described in Sec. 1: simply consider the largest quotient of $\mathcal{C}$ making invertible those morphisms (computable maps) $X \rightarrow Y$ that become computably invertible after the restriction to some subsets of $X$ and $Y$ with finite complements.

Another such structure is the class of Kolmogorov's orderings: total orders on $X$ defined by increasing Kolmogorov complexity with respect to various optimal enumerations.

Such orderings are not computable, but with respect to them all recursive functions, including admissible numberings, become functions of linearly bounded growth. 
I discuss this feature from the renormalization viewpoint in Sec. 3.

$B L O C K B$. This group consists of problems about interaction of computability with other Bourbaki sructures on $X$. An elementary example is the embedding $X=\mathbf{Q} \subset \mathbf{R}$ used in the theory of computable rational approximations to real numbers.

In this block, the Diophantine representability of enumerable subsets of $\mathbf{N}$ was the greatest discovery (Davis, Robinson, Putnam, Matiyasevich).

A very interesting and unexpected example of such interaction was elaborated in the work of A. Nabutovsky and S. Weinberger, (cf. [NaWe]), who have shown that the computational complexity can be used to display a highly irregular landscape of minima of natural differential-geometric functionals on the space of Riemannian metrics modulo diffeomorphisms.

Since path integration over such a space is one of the key tools of quantum gravity, this can become an important next meeting space between renormalization and computation.

Acknowledgements. I am very grateful to Cristian Calude, Leonid Levin, Mike Stay, Noson Yanofsky, who sent their remarks and suggestions incorporated in this draft of the article.

\section{Enriched programming methods with typing and parallelism: a categorical approach}

1.1. Preliminary remarks. We denote by $\mathbf{N}$ the set of natural numbers, and by $\mathbf{Z}^{+}$that of positive natural numbers. Usually $\mathbf{N}$ is taken as the basic set on which recursive functions are defined; in [Ma1] I used $\mathbf{Z}^{+}$having found it more convenient in a Diophantine context.

As a Bourbaki structure, both $\mathbf{Z}^{+}$and $\mathbf{N}$ are here (isomorphic) totally ordered sets, with a minimal element 1 (resp. 0) and successor function $\operatorname{suc}(x)$ : "the smallest $y$ such that $y>x "$, or $\operatorname{suc}(x)=x+1$ in the standard notation. In a sense, this is the minimal structure needed to define the set of partial recursive functions that are partial maps $\mathbf{Z}^{+} \rightarrow \mathbf{Z}^{+}$or, more generally $\left(\mathbf{Z}^{+}\right)^{a} \rightarrow\left(\mathbf{Z}^{+}\right)^{c}$. The remaining components of the definition (see e. g. [Ma1], V.2.1-2.4) are just the standard category-theoretic constructions in a fixed monoidal category of sets (ParSets, $\times$ ) with partial maps as morphisms and cartesian product: cf. [Ma3], 3.7.

However this total order structure is not invariant with respect to the structure that we will define below, in the sense that it is not preserved under the automor- 
phisms of this structure. For this reason, we avoid one of the standard categorifications of computation theory in which $\mathbf{N}$ is replaced by a "natural numbers object" $\mathcal{N}$ (of an abstract category), endowed with a morphism $\operatorname{suc}_{\mathcal{N}}: \mathcal{N} \rightarrow \mathcal{N}:$ this categorification unduly stresses the role of this total order and iteration $\operatorname{suc}_{\mathcal{N}} \circ \cdots \circ \mathrm{suc}_{\mathcal{N}}$ related to it.

Instead, we adopt the version advocated in [Ma2], that of a subcategory $\mathcal{C}$ of ParSets called Constructive Universe. In 1.2-1.8 below I collect the relevant formal definitions. Informal comments are relegated to 1.10 .

1.2. Objects. Objects of $\mathcal{C}$ will be called constructive worlds.

1.2.1. Definition. A constructive world $X$ is either a finite set, or an infinite set endowed with a nonempty set $N u m(X)$ of bijections $\nu: \mathbf{Z}^{+} \rightarrow X$, called admissible numberings, satisfying the following conditions:

(i) If $\nu_{1}, \nu_{2} \in \operatorname{Num}(X)$, then $\nu_{2}^{-1} \circ \nu_{1}$ is a total recursive bijection.

(ii) If $\nu \in N u m(X)$ and $f: \mathbf{Z}^{+} \rightarrow \mathbf{Z}^{+}$is a total recursive bijection, then $\nu \circ f \in N u m(X)$.

Elements of the constructive world $X$ are called constructive objects of the type $X$.

1.3. Morphisms. Let $X, Y$ be two constructive worlds. Morphisms $X \rightarrow Y$ are induced by partial recursive maps on their structure numberings. More precisely:

1.3.1. Definition. A morphism $X \rightarrow Y$ is partial map $f: D(f) \rightarrow Y$, where $D(f) \subset X$ a subset (possibly empty) satisfying the following conditions:

(i) If $X$ be infinite, $Y$ is finite, then for one (equivalently, any) admissible numbering $\nu: \mathbf{Z}^{+} \rightarrow X$ and any $y \in Y$, the set $\nu^{-1}\left(f^{-1}(y)\right)$ is recursive enumerable.

(ii) If $X$ and $Y$ are infinite, then for one pair (equivalently, any pair) admissible numberings $\nu_{X}: \mathbf{Z}^{+} \rightarrow X, \nu_{Y}: \mathbf{Z}^{+} \rightarrow Y$, the partial map $\nu_{Y}^{-1} \circ f \circ \nu_{X}: \mathbf{Z}^{+} \rightarrow \mathbf{Z}^{+}$ is a partial recursive function.

(iii) If $X$ is finite, $Y$ is infinite, any partial map is a morphism.

With the standard composition of partial maps, constructive worlds form a category $\mathcal{C}$, which we will call Constructive Universe. The set of morphisms $X \rightarrow Y$ will be denoted $\mathcal{C}(X, Y)$. Its subcategory consisting of infinite constructive worlds is equivalent to a very simple category consisting of one object, say $\mathbf{Z}^{+}$, and partial recursive maps as morphisms. In [He], such categories are called isotypical ones.

However, it is important to consider $\mathcal{C}$ as (bi)monoidal category, with two symmetric monoidal structures $\times$ (direct product) and $\amalg$ (coproduct, or disjoint union, see $[\mathrm{He}])$, connected by the standard coherence diagrams. 
These monoidal structures are induced by those in a small category of (unstructured) sets in which our constructive worlds lie, so that it suffices to specify some privileged numberings of disjoint sums and direct products. Moreover, it suffices to consider numberings that are bijective maps $\mathbf{Z}^{+} \rightarrow \coprod_{i=1}^{m} \mathbf{Z}^{+}$and $\mathbf{Z}^{+} \rightarrow\left(\mathbf{Z}^{+}\right)^{m}$.

For $\coprod_{i=1}^{m} \mathbf{Z}^{+}$, we simply assign to $m(k-1)+i$ the number $k$ in the $i$-th summand.

The cartesian product is more interesting, because there are several numberings that become privileged in the context of Kolmogorov complexity. TO BE CONTINUED ...

We will generally assume that $\mathcal{C}$ is closed with respect to the monoidal structures $\times$ and $\amalg$.

1.4. Constructive descriptions of morphisms. Fix two constructive worlds $X, Y$. Since the set of recursive maps $\mathcal{C}(X, Y)$ is not a constructive world, we may try to replace it by descriptions.

1.4.1. Definition. A constructive world of descriptions is a pair $(P(X, Y), F)$, where $P(X, Y)$ is an object of $\mathcal{C}$, and $F: P(X, Y) \times X \rightarrow Y$ is a morphism in $\mathcal{C}$, satisfying the following condition.

Let $p \in P(X, Y)$. Denote by $f_{p}$ the partial map $x \mapsto F(p, x) \in Y, x \in X$. Then each $f_{p}$ is a morphism $X \rightarrow Y$ in $\mathcal{C}$.

In other words, descriptions produce a set theoretic map $P(X, Y) \rightarrow \mathcal{C}(X, Y)$ constructively depending on $(p, x)$.

1.4.2. Translations. Let $(P(X, Y), F)$ and $(Q(X, Y), G)$ be two constructive worlds of descriptions. A translation (or compilation) method

$$
\operatorname{trans}_{P, Q}: P(X, Y) \rightarrow Q(X, Y)
$$

is an everywhere defined morphism in $\mathcal{C}$ such that for all $p \in P(X, Y)$, $\operatorname{trans}(p) \in$ $Q(X, Y)$ defines the same morphism $f_{p}: X \rightarrow Y$. In other words,

$$
G \circ\left(\operatorname{trans}_{P, Q} \times i d_{X}\right)=F \text {. }
$$

1.4.3. Universal descriptions. The world $(U(X, Y), W)$ is the world of universal descriptions, if for any other world of descriptions $(P(X, Y), F)$, there exists a translation morphism

$$
\operatorname{trans}_{P, U}: P(X, Y) \rightarrow U(X, Y)
$$


In particular, it can compute any (semi)computable function, in the sense that the family of maps $f_{u}: X \rightarrow Y, u \in U(X, Y)$ contain all morphisms in $\mathcal{C}$.

1.4.4. Complements. Among various constructive worlds of descriptions $P(X, Y)$ there exist ones with better properties than the general definition allows to guess. The terminology for them is rather unstable. We will say sometimes (see 2.6 below) that $P(X, Y)$ is the base of a family $f_{p}: X \rightarrow Y, p \in P(X, Y)$ of partial functions.

We will review below some relevant definitions and existence theorems. We consider separately four cases. $\mathbf{Z}^{+}$.

(i) If $X, Y$ are infinite, we may assume without losing generality that $X=Y=$

H. Rogers in [Ro] calls such a world of descriptions $U$ semi-effective, if it computes all morphisms (partial recursive functions), and he calls $U$ fully effective, if it is universal in the sense of 1.4.5.

An easy construction in [Ro] (example following Definition 3) shows that there are semi-effective descriptions that are not fully effective. A universal description world $U(X, Y)=\mathbf{Z}^{+}$(or rather $\mathbf{N}$ ) is also called a Gödel numbering in [Ro].

The main Theorem of [Ro] in our language implies that for any two universal description worlds (for infinite $X, Y$ ), there exist two mutually inverse translation isomorphisms between them: total recursive bijections, compatible with functions that these descriptions compute.

C. P. Schnorr in [Sch] considerably strengthens this result. Namely, he calls a universal description world $\left(U=\mathbf{Z}^{+}, W\right)$ an optimal Gödel numbering, if for any other world of descriptions $\left(P=\mathbf{Z}^{+}, F\right)$, there exists a translation morphism $t: P \rightarrow U$ which is a linearly bounded function $\mathbf{Z}^{+} \rightarrow \mathbf{Z}^{+}$. We will call such a description world simply optimal one.

Schnorr then proves that optimal descriptions exist, and for any two optimal description worlds (with infinite $X, Y$ ), there exist two mutually inverse linearly bounded translation isomorphisms between them.

Similar results hold in the case when only one of the worlds $X, Y$ is infinite. The general situation can be reduced to the case when the relevant finite world is one-element set; for simplicity, we will consider only this case.

(ii) The case $X=\{*\}$ is truly exceptional in the following sense: $\mathcal{C}(\{*\}, Y)$, that is " $Y$-valued recursive functions of zero variables", can be canonically identified with the set $Y$ and thus it is a constructive world. Nevertheless, the notions and 
main results of Rogers and Schnorr are applicable to this case as well and lead to a strengthening of the notion of Kolmogorov optimal enumeration of constructive objects of a given type.

(iii) In the case $Y=\{*\}, \mathcal{C}(X,\{*\})$ can be naturally identified with the set of all enumerable subsets of $X$, domains of partial recursive functions with one value. Gödel and optimal numberings of enumerable subsets also can be easily defined, and again the Rogers and Schnorr theorems are valid for them.

(iv) Finally, when both $X, Y$ are finite, the useful structurizations of descriptions are those of Boolean polynomials, circuits, etc. Many complexity problems are centered around polynomial time computations. Cf. [Ma2] for an introduction, that is close in style to this paper.

1.5. Enrichments of $\mathcal{C}$ over itself and programming methods. There is a well-known general notion of a category $C$ enriched over a monoidal category $(M, \otimes, I)$ where $I$ is an identity object.

Below, we will consider enrichments of $\mathcal{C}$ over $(\mathcal{C}, \times, I)$ where $I$ is a fixed oneelement constructive world. Products of empty families, such as $\mathbf{N}^{a}$ for $a=0$, are interpreted as $I$.

According to the general pattern, such an enrichment must consist of the following data. $\mathrm{ObC}$.

a) For each pair of constructive worlds $X, Y$, an "object of morphisms" $P(X, Y) \in$

b) For each triple of constructive worlds $X, Y, Z$, a "composition morphism" in $\mathcal{C}:$

$$
\circ: P(Y, Z) \times P(X, Y) \rightarrow P(X, Z)
$$

c) For each object $X$ of $\mathcal{C}$, an identity morphism

$$
\operatorname{id}_{X}: I \rightarrow P(X, X) .
$$

The standard axioms for morphisms in a category translate into requirements of commutativity of three classes of diagrams in $\mathcal{C}$ expressing properties of associativity of enriched composition, and left and right identities.

1.5.1. Definition. An enrichment of $\mathcal{C}$ over $(\mathcal{C}, \times, I)$ as above is called an enriched programming method and denoted $\mathcal{C}_{P}$, if the following additional data are given and axioms satisfied: 
For each pair of constructive worlds $X, Y$, a morphism in $\mathcal{C}$ is given

$$
F_{X, Y}: P(X, Y) \times X \rightarrow Y
$$

such that $P(X, Y)$ becomes a constructive world of descriptions in the sense of Definition 1.4.1. Thus, we have a family of set-theoretic maps

$$
\Phi_{X, Y}: P(X, Y) \rightarrow \mathcal{C}(X, Y): \quad p \mapsto f_{p}
$$

We will say that $p$ is a description, or a program, computing $f_{p}$.

Moreover, the following axioms must be satisfied:

(i) Morphisms (1.1) and (1.2) must be everywhere defined (total recursive) maps. (For (1.2), this means simply that they are non-empty maps).

(ii) Compositions (1.1) must be compatible with the compositions of morphisms in $\mathcal{C}$ :

$$
f_{p \circ q}=f_{p} \circ f_{q} .
$$

(iii) Element $\operatorname{id}_{X}(I) \in P(X, X)$ must be a description of the "copying" program: mapping $x$ to $x$.

(The latter should not be mixed with the "cloning" program computing the diagonal map $X \rightarrow X \times X, x \mapsto(x, x)$.)

Informally speaking, we have a functor

$$
\Phi_{P}: \mathcal{C}_{P} \rightarrow \mathcal{C}
$$

identical on objects and mapping a program to the function that this program computes.

The most important feature of this formalism consists in an explicit and systematic inclusion of composition of programs into our formalism: this is a key requirement for all Hopf algebra renormalization schemes.

Finally, a remark on terminology: we use the word program as a synonym of "description of a method to compute a given function". Input of such a program is a specific value of the argument of this function, output is the value of the function. This praxis should not be confused with the one used in the theory of Turing machines, where programs are often understood as our inputs: the initial binary string on the tape. 
1.6. A (uni)versal enrichment. An enrichment $\mathcal{C}_{U}$ as above is called (uni)versal one, if programs from $U$ compute all partial recursive maps, and moreover, if for each $\mathcal{C}_{P}$, there is a functor between enriched categories

$$
\Psi: \mathcal{C}_{P} \rightarrow \mathcal{C}_{U}
$$

identical on objects, with total recursive maps

$$
\Psi_{X, Y)}: P(X, Y) \rightarrow U(X, Y)
$$

such that $\Psi_{X, Y}(p)$ for each $p$ computes the same function as $p$. In other words, we have

$$
\Phi_{U} \circ \Psi=\Phi_{P}
$$

Intuitively, we want the following properties of $U$ as a programming method, that are somewhat stronger than those in 1.4.

a) It can compute any (semi)computable function.

b) For each other programming method $P$, there must exist a computable (on the world of $P$-programs) translation of $P$-programs into $U$-programs, computing the same functions.

c) The translation must be compatible with composition of programs and copying/identity programs (functorality of $\Psi$ ).

1.7. Coproducts and typing. Coproducts (disjoint sums) admit the most straightforward interpretation in the contexts, where computer scientists speak about typing. In the simplest situation, a program $p \in P(X \coprod Y, Z)$ accepts inputs of either type $X$ or $Y$, and produces outputs of type $Z$.

Iterated application of similar interpretations, as far as I can judge, can be used in all contexts where the notion of typing is essential.

1.8. Products and parallelism. Let $X_{i}, Y_{i}, i=1, \ldots, n$ be constructive worlds. Then the map

$$
\begin{gathered}
\pi: \mathcal{C}\left(X_{1}, Y_{1}\right) \times \mathcal{C}\left(X_{2}, Y_{2}\right) \times \cdots \times \mathcal{C}\left(X_{n}, Y_{n}\right) \rightarrow \mathcal{C}\left(X_{1} \times X_{2} \times \cdots \times X_{n}, Y_{1} \times Y_{2} \times \cdots \times Y_{n}\right), \\
\pi\left(f_{1}, \ldots, f_{n}\right)\left(x_{1}, \ldots, x_{n}\right):=\left(f_{1}\left(x_{1}\right), \ldots, f_{n}\left(x_{n}\right)\right)
\end{gathered}
$$

defines families of computations with independent inputs/outputs that can be implemented parallely. This can be generalized to programming methods as follows. 
1.8.1. Definition. Let $P$ be an enriched programming method. We will say that $P$ admits unrestricted parallelism, if the following additional structure is given.

Let $\left\{X_{i}\right\},\left\{Y_{i}\right\}, i=1, \ldots, n$, be any finite family $\sigma$ of constructive worlds. We must be given maps

$$
\pi_{\sigma}: \prod_{i=1}^{n} P\left(X_{i}, Y_{i}\right) \rightarrow P\left(\prod_{i=1}^{n} X_{i}, \prod_{i=1}^{n} Y_{i}\right),
$$

that are lifts of (1.7). These maps must be equivariant with respect to the natural action of the symmetric group $S_{n}$ permuting subscripts at both sides.

1.9. Basic example: flowcharts. Flowcharts defined in [Ma3] form a convenient context for constructing enriched progamming methods with unrestricted parallelism. One example of of such a method is the world $P$, computing primitive recursive functions, described im [Ma3], Definition 2.11. This definition uses the ideas of N. Yanofsky paper [Ya].

Additional work remains to be done in order to produce a manageable construction of an universal enrichment with unrestricted parallelism.

1.10. Comments: constructive worlds and admissible numberings. Technically speaking, conditions (i) and (ii) of Definition 1.2.1 together mean that $\operatorname{Num}(X)$ forms a principal homogeneous space over the group of total recursive permutations of $\mathbf{Z}^{+}$which we may denote $S_{\mathbf{Z}^{+} \text {,rec }}$, as an infinite analog of nite symmetric groups. Hence the whole $N u m(X)$ can be reconstructed from any one numbering in this set. Usually there are some simplest, or privileged numberings, with which one mostly works, such as the numbering of binary words $w \in\{0,1\}^{\mathbf{N}}$ used in [CalSt1]: bin $^{-1}: w \mapsto \overline{1 w}$, where the line over a binary word means that it should be treated as a natural number given by its binary digits.

The idea of privileged numberings is essential especially when one deals with polynomial time, or more general "feasible" computations. In order to accommodate this idea, we can strengthen Definition 1.2.1 in the following way. Consider the smaller "symmetric group" $S_{\mathbf{Z}^{+}, \text {pol }}$ of total recursive permutations that are polynomial time computable together with their inverses. Define the structure of polynomial time constructive world $X$ by a set $N u m p o l(X)$ forming a principal homogeneous space over the group $S_{\mathbf{Z}^{+}, \text {pol }}$.

An (often implicit) part of contemporary philosophy around Turing's Thesis consists in postulating that whenever we can informally speak about algorithms and (semi) computable maps between two constructive worlds, we always can produce in 
the context of this discussion admissible numberings that are informally algorithmic and transform informal (semi)computable maps into (partial) recursive functions.

In any case, starting with such a class of numberings of $X$, we want to stress that we study notions that are either invariant, or behave in a controlled way under the action of the group $S_{X, r e c}$.

We add a few more remarks.

Sometimes, a constructive world $X$ itself is an unstructured set, in the sense that the only relevant structure on it is given by its set of admissible numberings. The typical example is a world $A$ that in further constructions may serve as an alphabet. In this case, a numbering dening the whole $N u m(A)$ usually is introduced ad hoc. But in most applications, $X$ itself consists of certain sets (often finite and/or considered only up to isomorphism, or even organized into a category) endowed with a certain fixed Bourbaki structure, such as:

(a) Finite words in an alphabet $A$.

(b) Finite graphs up to an isomorphism.

(c) Finite groups.

In such cases, the privileged numberings ("encodings") generating the whole $\operatorname{Num}(X)$ are supposed to interact with this structure in such a way that the number of a constructive object can be "algorithmically calculated" when we know this object as an instance of this structure, and vice versa, this instance must be algorithmically reconstructible from the number. It is only very rarely that such encodings can translate well the basic relations, composition laws etc., involved in the definition of the structure. This is one reason to formulate models of computation directly in terms of this structure: one can recall Church's lambda-calculus, Kolmogorov-Uspenski's graphs and Gács-Levin causal nets (cf. [GaLe]).

On the other hand, such a simple task as the choice of a privileged numbering of $\mathbf{Z}^{+} \times \mathbf{Z}^{+}$(upon which one of the monoidal structures on $\mathcal{C}$ is based) can lead to quite interesting constructions when this choice is related, for example, with complexity estimates: see our discussion in 2.7-2.10 below using L. Levin's norms for definitions of such numberings.

Notice that instances of the constructive worlds of causal nets studied in [GaLe] are themselves categories, and the study of interaction of computability with symmetries of the respective constructive objects reveals interesting new phenomena.

We want to argue that our categorical framework suggests other possibilities to avoid too close attention to the elementary steps of computation. In particular, the "categorical Church Thesis" admits a wonderfully succinct expression: 
The category $\mathcal{C}$ is defined uniquely up to equivalence.

An important complication and variation of the theme of admissible numberings arises, when a structure $S$ that we want to treat "constructively" is thus imposed on eventually innite sets. In such cases the relevant constructive objects are often not the structures themselves, but their finite descriptions: a group might be given by generators and relations, an affine scheme over $\mathbf{Z}$ by its equations etc.

The usual complication with descriptions is that many descriptions can produce one and the same (or canonically isomorphic) Bourbaki structure, and the relevant equivalence relation on the set of descriptions can be undecidable, or even not recursively enumerable. This is precisely the case of the structure constituted by recursive functions themselves, which is our main motivation for introducing enrichments as in Definitions 1.5.1 and 1.8.1.

\section{Cut-off regularization and Anytime Algorithms}

2.1. Cut-off regularization. In Quantum Field Theory, cut-off regularization schemes have the following typical structure. The relevant Feynman integrals, say, in momentum space, may diverge when momentum becomes large (resp. small). In this case, the formal integral in question $I$ is replaced by the finite integral $I_{P}$ taken over momenta $p \leq P:=p_{\text {cutoff }}$ (resp. $p \geq P:=p_{\text {cutoff }}$ ). The behavior of $I_{P}$ as $P \rightarrow \infty$ (resp. $P \rightarrow 0$ ) is then studied, and physical information is extracted from the behavior of the polar part, or regular part, of $I_{P}$.

In computer science based upon Turing machines and/or recursive functions, the natural "divergence" occurs in space-time: a computation uses discrete memory (space) and runtime. A typical example of such a divergence is the infinite runtime of a Turing machine computing a partial recursive function $f$ at an input (program) $x$ which is outside the definition domain of $f$.

Application-oriented computer scientists, of course, recognize the practical necessity of time cut-offs, accompanied by sober estimates of quality of outputs. Systematic work on this problem resulted in the notion of "Anytime Algorithms", cf. [GrZi]. The usefulness of composition and exploiting parallelism was stressed in $[\mathrm{RuZi}]$.

In a stimulating paper [CalSt1], Ch. Calude and M. Stay addressed the problem of cut-off of runtime theoretically, and designed meaningful quantitative characteristics of such a cut-off.

More precisely, let $f$ be a partial recursive function ("a morphism of constructive worlds $X \rightarrow Y$ ") as above, and $F$ its description, a program calculating it. Computation time (or runtime) is another partial recursive function, with the same 
domain $D(t(F))=D(f) \subset X$ and target $\mathbf{Z}^{+}$, whose precise definition depends on the details of the implied choice of our programming method.

For a Turing machine $F, t(F)(x)$ is the number of steps required to halt and print $f(x)$ on the tape, if $x \in D(f)$. One can similarly define another partial recursive function, "memory volume" $m(F): X \rightarrow \mathbf{Z}^{+}$such that for $x \in D(f), m(F)(x)$ is the minimal length of tape required to compute $f(x)$. Here $W$ is the constructive world of binary words $\{0,1\}^{\mathbf{Z}^{+}}$. Yet another partial recursive function, $s(F)$, is the sum total of lengths of filled parts of the tape over all steps of computation. Notice that the settling function of Soare ([So], Definition 8.2), which is essentially $\max \{t(F)(y) \mid y<x, y \in D(f)\}$, generally is not partial recursive, but some of the inequalities stated below, such as (2.3), are valid for it as well.

One can define natural analogs of functions $t(F), m(F)$, and $s(F)$ for rather general normal programming methods $F$, discussed in [Ma2] and Chapter IX of the new edition of [Ma1].

Returning to [CalSt1], we will first of all show that some of the basic results of that paper related to cut-offs, admit a straightforward reformulation in such a way that they become applicable and true for any partial recursive function, including, of course, $t(F), m(F)$, and $s(F)$.

This naturally raises a question, what is so specific about $t(F), m(F)$, and $s(F)$. We will treat this question in 2.6 below in the context of categorification developed in Sec. 1, and will show that this provides some meaningful insights about these measures of processes of computation.

2.2. Complexity. I will first recall the definition and properties of the Kolmogorov ("exponential", or "program") complexity $C_{u}: \mathbf{Z}^{+} \rightarrow \mathbf{Z}^{+}$, cf. [Ma1], VI.9. In [CalSt1] it is called the natural complexity and denoted $\nabla_{U}$ or simply $\nabla$.

This complexity measure is defined with respect to a partial recursive function $u: \mathbf{Z}^{+} \rightarrow \mathbf{Z}^{+}$which is surjective:

$$
C_{u}(x):=\min \{y \mid u(y)=x\}
$$

This function $u$ is an arbitrary element of the set of Kolmogorov, or Gödel, optimal functions, representatives of which can be effectively constructed: cf. [Ro], [Sch] and [Ma1]. Optimality implies that for any other partial recursive $v: \mathbf{Z}^{+} \rightarrow \mathbf{Z}^{+}$, there exists a constant $c_{u, v}>0$ such that for all $x, C_{u}(x) \leq c_{u, v} C_{v}(x)$. (The right hand side is interpreted as $\infty$, if $x$ is not in the range of $v$ ). 
It follows that another choice of optimal function replaces $C_{u}$ by a function $C_{u^{\prime}}=2^{O(1)} C_{u}$. We will say that two such functions belong to the same bounded equivalence class.

Moreover, as we have discussed above, the definition of the complexity of integers can be extended to the definition of complexity of partial recursive functions of any fixed number of variables $m$, as in VI.9.1 of [Ma1]. This requires a choice of Kolmogorov optimal recursive function of $m+1$ variables. We have then the following simple result (omitting the subscripts at $C$ specifying the choices of optimal families, and denoting by $c$ with subscripts various constants depending on these choices as well):

2.2.1. Proposition. For any partial recursive function $f: \mathbf{Z}^{+} \rightarrow \mathbf{Z}^{+}$and $x \in D(f)$ we have

$$
C(f(x)) \leq c_{f} C(x) \leq c_{f}^{\prime} x,
$$

If $f$ and $x \in D(f)$ are allowed to vary, we have

$$
C(f(x)) \leq c C(f) C(x) \log (C(f) C(x)) .
$$

In particular, if $f$ is a total recursive permutation, then complexities of $x$ and $f(x)$ are bounded equivalent. It follows that we can define the complexity function, up to bounded equivalence, $C: X \rightarrow \mathbf{Z}^{+}$for any infinite constructive world $X$ : choose an admissible numbering $\nu: \mathbf{Z}^{+} \rightarrow X$ and put $C(x):=C_{u}\left(\nu^{-1}(x)\right)$ for some optimal $u$.

2.3. Runtimes according to [CalSt1]. Proposition 2.2.1 is a special case of Proposition 9.6 in [Ma1], VI.9. In turn, it implies as special cases the inequality (2) and Theorem 4 of [CalSt1].

In order to see this, one has simply to compare terminology and notation.

[CalSt1] deals with the complexity $C$ (their $\nabla=\nabla_{U}$ ) of binary words, that reduces to the complexity of integers via the admissible numbering denoted bin in [CalSt1]. It is defined via a "universal Turing machine" $U$, which in our language is a programming method computing one of the Kolmogorov optimal functions $u$. Consider the partial recursive function $x \mapsto t(U)(x)$ : runtime of $U$ at the argument $x \in \mathbf{Z}^{+}$. The inequality (2) of [CalSt1] in our notation can be rewritten as

$$
C(t(U)(x)) \leq c x
$$


which is our (2.1) for $f=t(U)$. The same inequality is valid for $m(U), s(U)$, but also for $t(F), m(F), s(F)$ for any $F$, and even for Soare's settling functions: see section 2.1 above.

2.4.1. Growth of recursive functions and algorithmic randomness. The central argument of [CalSt1] is based upon two statements:

a) The runtime of the Kolmogorov optimal program at a point $x$ of its definition domain is either $\leq c x^{2}$, or is not "algorithmically random" (Theorem 5 of [CalSt1]).

b) "Algorithmically random" integers have density zero for a class of computable probability distributions.

This last statement justifies the time cut-off prescription which is the main result of [CalSt1]:

if the computation on the input $x$ did not halt after $c x^{2}$ Turing steps, stop it, decide that the function is not determined at $x$, and proceed to $x+1$.

Proposition 2.5.1 below somewhat generalizes the statement a).

2.5. Randomness and growth. Consider a pair of functions $\varphi, \psi: \mathbf{R}_{>0} \rightarrow$ $\mathbf{R}_{>0}$ satisfying the following conditions:

a) $\varphi(x)$ and $\frac{x}{\varphi(x)}$ are strictly increasing starting with a certain $x_{0}$ and tend to infinity as $x \rightarrow \infty$.

b) $\psi(x)$ and $\frac{\psi(x)}{x \varphi(\psi(x))}$ are increasing and tend to infinity as $x \rightarrow \infty$.

The simplest examples are $\varphi(x)=\log (x+2), \psi(x)=(x+1)^{1+\varepsilon}, \varepsilon>0$.

In our context, $\varphi$ will play the role of a "randomness scale". Call $x \in \mathbf{Z}^{+}$ algorithmically $\varphi$-random, if $C(x)>x / \varphi(x)$. The second function $\psi$ will then play the role of associated growth scale.

2.5.1. Proposition. Let $f$ be a partial recursive function. Then for all sufficiently large $x$ exactly one of the following alternatives holds:

(i) $x \in D(f)$, and $f(x) \leq \psi(x)$.

(ii) $x \notin D(f)$.

(iii) $x \in D(f)$, and $f(x)$ is not algorithmically $\varphi$-random.

Proof. We must only check that if $x \in D(f)$ and $f(x)>\psi(x)$, then $f(x)$ is not algorithmically $\varphi$-random, that is

$$
C(f(x)) \leq \frac{f(x)}{\varphi(f(x))} .
$$


In fact, in view of (2.1),

$$
C(f(x)) \leq c x
$$

for some constant $c$ (depending on $u$ and $f$ ). Furthermore, for sufficiently large $x$, in view of $2.5 \mathrm{~b}$ ), we have

$$
c x \leq \frac{\psi(x)}{\varphi(\psi(x))} \leq \frac{f(x)}{\varphi(f(x))}
$$

Clearly, (2.5) and (2.6) imply (2.4).

2.6. Cost estimate functions. Since, as we argued, the randomness/growth alternative holds for arbitrary recursive functions, not only for runtimes and alike, we will briefly discuss specific properties of runtimes, considered from the perspective of categorification, explained in sec. 1.

Let $P$ be an enriched programming method, as in Definition 1.5.1. We will say that a partial function $\delta: P(X, Y) \times X \rightarrow \mathbf{Z}^{+}$is a cost estimate function, if the following conditions are satisfied:

(i) $\delta$ is partial recursive (morphism in $\mathcal{C}$ ), and $D(\delta)=\left\{(p, x) \mid x \in D\left(f_{p}\right)\right\}$.

(ii) $\delta(p \circ q, x)=\delta(q, x)+\delta(p, q(x))$ whenever both sides are defined.

The requirement (i) is natural, because the "run-cost" of computation (time, maximum storage size) must be computable in terms of cost increments required at each step. The requirement (ii) then expresses the additivity of such increments. We may, or may not, ascribe a non-zero cost to the program calculating identical function ("data transfer").

Requirement (i), complemented by the requirement of the decidability of the graph of $\delta$, constitute two axioms due to M. Blum. This latter property has a clear intuitive meaning as well.

Finally, if our cost estimate function refers to time only, and we allow the unrestricted parallelism, the following property is natural. Using notation (1.8), we must have

(iii) $\delta\left(\pi_{\sigma}\left(p_{1}, \ldots, p_{n}\right),\left(x_{1}, \ldots, x_{n}\right)\right)=\max \left(\delta\left(p_{1}, x_{1}\right), \ldots, \delta\left(p_{n}, x_{n}\right)\right)$.

2.7. Constants related to Kolmogorov complexity estimates. Since inequalities (2.1), (2.2), and their extensions are often useful, we will say a few words about their computability. 
As in [Ma3], VI.9, we call partial maps $\left(\mathbf{Z}^{+}\right)^{m} \rightarrow \mathbf{Z}^{+}, m \geq 0, m$-functions. A Kolmogorov optimal family of $m$-functions $u\left(x_{1}, \ldots, x_{m} ; k\right), k \in \mathbf{Z}^{+}$, is produced from two inputs: $U$.

(a) A fully effective (in the sense of Rogers, cf. 1.4.4) family of $(m+1)$-functions

(b) A recursive embedding $\theta: \mathbf{Z}^{+} \times \mathbf{Z}^{+} \rightarrow \mathbf{Z}^{+}$with decidable image, satisfying a linear growth condition

$$
\theta(k, j) \leq k \cdot \varphi(j)
$$

where $\varphi: \mathbf{Z}^{+} \rightarrow \mathbf{Z}^{+}$an appropriate function.

Having made these choices, we put

$$
u\left(x_{1}, \ldots, x_{m} ; k\right):=U\left(x_{1}, \ldots, x_{m} ; \theta^{-1}(k)\right) .
$$

Then for any other family $v$ of $m$-functions $v$ with base $\mathbf{Z}^{+}$and each $m$-function $f$, we have the inequality

$$
C_{u}(f) \leq c_{u, v} C_{v}(f)
$$

with

$$
c_{u, v}:=\varphi\left(C_{U}(v)\right) .
$$

(cf. [Ma1], VI.9.4).

Clearly, (2.1) is a special case of (2.9). An effective estimate of (2.10) from above will be assured, if $\varphi$ is computable and increasing, and if, knowing a $P$-description of $v$, we can find some member of the family $U$ coinciding with $v$. The latter, in turn, is automatic, if $P$ is supplied with a translation morphism $\operatorname{trans}_{P, U}$.

We will now discuss numberings $\theta$.

2.8. Slowly growing numberings. Let $R=\left(R_{k} \mid k \in \mathbf{Z}^{+}\right)$be a sequence of positive numbers tending to infinity with $k$. For $M \in \mathbf{Z}^{+}$, put

$$
V_{R}(M):=\left\{(k, l) \in\left(\mathbf{Z}^{+}\right)^{2} \mid k R_{l} \leq M\right\} .
$$

Clearly,

$$
\operatorname{card} V_{R}(M) \leq \sum_{l=1}^{\infty}\left[\frac{M}{R_{l}}\right]<\infty,
$$

where $[a]$ denotes the integral part of $a$. 
We have

$$
V_{R}(M) \subset V_{R}(M+1),\left(\mathbf{Z}^{+}\right)^{2}=\cup_{M=1}^{\infty} V_{R}(M) .
$$

Therefore we can define a bijection $N_{R}: \mathbf{Z}^{+} \rightarrow\left(\mathbf{Z}^{+}\right)^{2}$ in the following way: $N_{R}(k, l)$ will be the number of $(k, l)$ in the total ordering $<_{R}$ of $\left(\mathbf{Z}^{+}\right)^{2}$ determined inductively by the following rule: $(i, j)<_{R}(k, l)$ iff one of the following alternatives holds:

(a) $i R_{j}<k R_{l}$;

(b) $i R_{j}=k R_{l}$ and $j<l$;

2.9. Proposition. The numbering $N_{R}$ is well defined and has the following property: all elements of $V_{R}(M+1) \backslash V_{R}(M)$ have strictly larger numbers than those of $V_{R}(M)$. Moreover:

(i) If each $R_{l}$ is rational, or computable from above, then $N_{R}$ is computable (total recursive). then

(ii) If the series $\sum_{l=1}^{\infty} R_{l}^{-1}$ converges and its sum is bounded by a constant $c$,

$$
N_{R}(k, l) \leq c\left(k R_{l}+1\right) .
$$

(iii) If the series $\sum_{l=1}^{\infty} R_{l}^{-1}$ diverges, and

$$
\sum_{l=1}^{M} R_{l}^{-1} \leq F(M)
$$

for a certain increasing function $F=F_{R}$, then

$$
N_{R}(k, l) \leq\left(k R_{l}+1\right) F\left(k R_{l}+1\right) .
$$

Proof. The first statements are an easy exercise. For (2.13) and (2.14), notice that if $M$ is the minimal value for which $(k, l) \in V_{R}(M)$, we have $M-1<k R_{l} \leq M$ and

$$
N_{R}(k, l) \leq \operatorname{card} V_{R}(M),
$$

and in the case (ii) we have from (2.12)

$$
\operatorname{card} V_{R}(M) \leq \sum_{m=1}^{\infty} M R_{m}^{-1} \leq c\left(k R_{l}+1\right) .
$$


Similarly, in the case (iii) we have

$$
\operatorname{card} V_{R}(M) \leq M \sum_{m=1}^{M} R_{m}^{-1} \leq\left(k R_{l}+1\right) F\left(k R_{l}+1\right)
$$

2.10. L. Levin's norms. From (2.13) one sees that any sequence $\left\{R_{l}\right\}$ with converging $\sum_{l} R_{l}^{-1}$ can be used in order to construct the bijection $\mathbf{Z}^{+} \times \mathbf{Z}^{+} \rightarrow \mathbf{Z}^{+}$, $(k, l) \mapsto N_{R}(k, l)$ linearly growing wrt $k$. Assume that it is computable and therefore can play the role of $\theta$ in (2.7) (b).

In this case, for any integer $M$ the set $V_{R}(M)$ must be decidable. It follows that for any $l$, the set of rational numbers $k / M \leq r_{l}:=R_{l}^{-1}$ is decidable.

Even if we weaken the last condition, requiring only recursivity of the set $k / M \leq$ $r_{l}$ (i. e. asking each $r_{l}$ to be computable from below), the convergence of $\sum_{l} r_{l}$ implies that there is a universal upper bound (up to a constant) for such $r_{l}$. Namely, let $C P$ be the prefix Kolmogorov complexity on $\mathbf{Z}^{+}$defined with the help of a certain optimal prefix enumeration.

2.10.1. Proposition. ([Le]). For any sequence of computable from below numbers $r_{l}$ with convergent $\sum_{l} r_{l}$, there exists a constant $c$ such that for all $l$, $r_{l} \leq c \cdot C P(l)^{-1}$

More generally, L. Levin constructs in this way a hierarchy of complexity measures associated with a class of abstract norms, functionals on sequences computable from below.

\section{Regularization and renormalization of the Halting Problem}

3.1. Introduction. In this section, we devise simple regularization/renormalization schemes tailored to fit the halting problem. The general structure of such a scheme is sketched in [Ma3], subsection 0.2. It involves the following components.

(a) Deforming the Halting Problem. At this step, we transform the problem of recognizing, whether a number $k \in \mathbf{Z}^{+}$belongs to the definition domain $D(f)$ of a partial recursive function $f$, to the problem, whether an analytic function $\Phi(k, f ; z)$ of a complex parameter $z$ has a singularity (in our case, a pole) at $z=1$.

In fact, using an idea from quantum computing, we may reduce the case of arbitrary $f$ to the case of a partial recursive permutation $\sigma=\sigma_{f}: D(\sigma) \rightarrow D(\sigma)$ of its definition domain, and construct $\Phi(k, \sigma ; z)$ for such permutations. This reduction is described in [Ma3], subsections $3.6-3.8$. 
(b) Choosing a minimal subtraction algebra. Our choice of an appropriate minimal subtraction algebra (see the definition in [Ma3], 4.2) is based on the established properties of functions $\Phi(k, \sigma ; z)$ : cf. Proposition 3.5 below.

Namely, let $\mathcal{A}_{+}$be the algebra of analytic functions in $|z|<1$, continuous at $|z|=1$. It is a unital algebra; we endow it with augmentation $\varepsilon_{\mathcal{A}}: \Phi(z) \mapsto \Phi(1)$.

Put $\mathcal{A}_{-}:=(1-z)^{-1} \mathbf{C}\left[(1-z)^{-1}\right]$. Finally, let $\mathcal{A}:=\mathcal{A}_{+} \oplus \mathcal{A}_{-}$.

Now we can use Theorem 4.4.1 of [Ma3] in renormalization schemes, involving a connected filtered Hopf algebra $\mathcal{H}$ (cf. [E-FMan], sec. 2.5, Theorem 1, and [Ma3], 4.1). It remains to indicate, which Hopf algebras and their $\mathcal{A}$-characters will be involved in this game.

(c) Hopf algebra of an enriched programming method. A class of such algebras is described in [Ma3], subsections $3.3-3.4$. This construction explicitly refers to flowcharts, however, it can be readily modified and generalized to enriched programming methods $P$ in the sense of Definition 1.5.1.

Basically, $\mathcal{H}=\mathcal{H}_{P}$ is the symmetric algebra, spanned by isomorphism classes $[p]$ of certain descriptions belonging to, say, $P\left(\mathbf{Z}^{+}, \mathbf{Z}^{+}\right)$. Comultiplication in $\mathcal{H}_{P}$ is dual to the composition of descriptions:

$$
\Delta([p]):=\sum_{q, r \mid q \circ r=p}[r] \otimes[q]
$$

(Recall that the composition of descriptions is associative).

In order to ensure finiteness of the right hand side of (3.1) and to produce a Hopf filtration, we must postulate in addition existence of a "size function" on descriptions. The simplest properties of such a function $p \mapsto|p|$ that will serve our goal, are finiteness of the set of descriptions of bounded size, and additivity

$$
|q \circ r|=|q|+|r|
$$

For a concrete example, see [Ma3], subsection 3.4.

(d) Characters, corresponding to the halting problem. Finally, assume that we have constructed $\mathcal{H}_{P}$ and $\Phi(k, f ; z)$ as above. Then the character $\varphi_{k}: \mathcal{H}_{P} \rightarrow \mathcal{A}$ (cf. [Ma3], 4.4) corresponding to the halting problem at a point $k \in \mathbf{Z}^{+}$for the partial recursive function computable with the help of a description $p \in P\left(\mathbf{Z}^{+}, \mathbf{Z}^{+}\right)$, is defined as follows:

$$
\varphi_{k}([p]):=\Phi(k, f ; z) \in \mathcal{A} \text {. }
$$


As soon as this definition is adopted, the machinery and philosophy of Hopf renormalization and Birkhoff decomposition ([Ma3], Theorem 4.4.1) becomes applicable to the classical halting problem.

Perhaps, it will be even more relevant for quantum computation schemes based upon infinite-dimensional Hilbert spaces.

3.2. The simplest construction. Let $f: \mathbf{Z}^{+} \rightarrow \mathbf{Z}^{+}$be a partial recursive function. Consider its extension $\bar{f}: \mathbf{N} \rightarrow \mathbf{N}$ defined as follows: $\bar{f}(x)=f(x)$ if $x \in D(f)$ and $f(x)=0$ otherwise.

Put

$$
\Psi(k, f ; z):=\sum_{n=0}^{\infty} \frac{z^{n}}{(1+n \bar{f}(k))^{2}} .
$$

3.2.1. Proposition. (i) If $k \notin D(f)$, then

$$
\Psi(k, f ; z)=\frac{1}{1-z} .
$$

(ii) If $k \in D(f)$, then $\Psi(x, \sigma ; z)$ is the Taylor series of a function analytic at $|z|<1$ and continuous at the boundary $|z|=1$. The value $\bar{f}(k)=f(k)$ can be uniquely reconstructed from $\Psi$, for example

$$
f(k)=\sqrt{\left.\frac{d z}{d \Psi}\right|_{z=0}}-1 .
$$

The proof is obvious.

Actually, formula (3.3) can be seen in its natural context if one invokes the general prescription of reducing any function to a permutation, borrowed from the theory of quantum computation.

I will briefly recall this prescription now following [Ma3], subsections 3.6 - 3.8.

3.3. Reduction of the general halting problem to the recognition of fixed points of permutations. Start with a partial recursive function $f: X \rightarrow X$ where $X$ is an infinite constructive world. Extend $X$ by one point, i. e. form $X \coprod\left\{*_{X}\right\}$. Choose a total recursive structure of an additive group without torsion on $X \coprod\left\{*_{X}\right\}$ with zero $*_{X}$. Extend $f$ to the everywhere defined (but generally uncomputable) function $g: X \coprod\left\{*_{X}\right\} \rightarrow X \coprod\left\{*_{X}\right\}$, by

$$
g(y):=*_{X} \text { if } y \notin D(f) .
$$


Define the map

$$
\tau_{f}:\left(X \coprod\left\{*_{X}\right\}\right)^{2} \rightarrow\left(X \coprod\left\{*_{X}\right\}\right)^{2}
$$

by

$$
\tau_{f}(x, y):=(x+g(y), y) \text {. }
$$

Clearly, it is a permutation. Since $\left(X \coprod\left\{*_{X}\right\},+\right)$ has no torsion, the only finite orbits of $\tau_{f}^{\mathbf{Z}}$ are fixed points.

Moreover, the restriction of $\tau_{f}$ upon the recursive enumerable subset

$$
D\left(\sigma_{f}\right):=\left(X \coprod\left\{*_{X}\right\}\right) \times D(f)
$$

of the constructive world $Y:=\left(X \coprod\left\{*_{X}\right\}\right)^{2}$ induces a partial recursive permutation $\sigma_{f}$ of this subset.

Since $g(y)$ never takes the zero value $*_{X}$ on $y \in D(f)$, but always is zero outside it, the complement to $D\left(\sigma_{f}\right)$ in $Y$ consists entirely of fixed points of $\tau_{f}$.

Thus, the halting problem for $f$ reduces to the fixed point recognition for $\tau_{f}$.

3.4. Permutations with bounded shift. The formula (3.3) can be generalized as follows.

3.4.1. Definition. Let $\sigma$ be a permutation of $\mathbf{Z}^{+}, k \in \mathbf{Z}^{+}$. We say that $\sigma$ has $a$ bounded shift at $k$ if there exist constants $a, b, c$ (depending on $\sigma$ and $k$ ) such that for all $n \in \mathbf{Z}$,

$$
c \cdot|n+a| \leq \sigma^{n}(k) \leq c \cdot|n+b| \cdot
$$

3.4.2. Lemma. If $\sigma$ has bounded shift at $k$, then the $\sigma^{\mathbf{Z}}$-orbit of $k$ is infinite, and for any $m \neq 0$ and any point of this orbit $l, \sigma^{m}$ has bounded shift at $l$.

Proof. Let $l=\sigma^{d}(k)$. From (3.3) we get

$$
c \cdot|m n+d+a| \leq \sigma^{m n}(l)=\sigma^{m n+d}(k) \leq c \cdot|m n+d+b|
$$

that is

$$
c|m| \cdot\left|n+\frac{d+a}{m}\right| \leq\left(\sigma^{m}\right)^{n}(l) \leq c|m| \cdot\left|n+\frac{d+b}{m}\right| .
$$

This inequality has the same form as (3.7), with different constants. 
3.5. Proposition. Let $\sigma$ be a permutation of $\mathbf{Z}^{+}, k \in \mathbf{Z}^{+}$. Put

$$
\Psi(k, \sigma ; z):=\sum_{n=1}^{\infty} \frac{z^{n}}{\left(\sigma^{n}(k)\right)^{2}} .
$$

Then we have:

(i) If $\sigma^{\mathbf{Z}}$-orbit of $k$ is finite, then $\Phi(\sigma, x ; z)$ is a rational function in $z$ whose all poles are of the first order and lie at roots of unity.

(ii) If this orbit is infinite, and $\sigma$ has bounded shift at (any point of) this orbit, then $\Phi(\sigma, k ; z)$ is the Taylor series of a function analytic at $|z|<1$ and continuous at the boundary $|z|=1$.

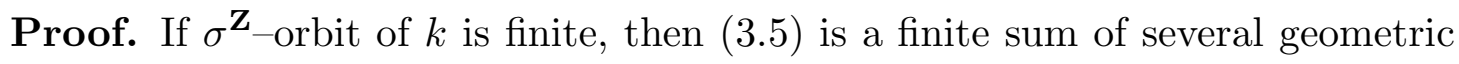
progressions each of each sums to a rational function of the type

$$
\text { const } \cdot \frac{z^{k}}{1-z^{l}}
$$

Otherwise, because of (3.7) we get a series absolutely converging for $|z| \leq 1$. This proves our statement.

3.6. The Kolmogorov order. Many interesting $\sigma$, such as total recursive permutations, are not permutations of bounded shift. To cope with this situation, we will (uncomputably) reorder $\mathbf{Z}^{+}$, and show, that after this reordering, all partial recursive functions and permutations corresponding to them will satisfy a version of bounded shift property, allowing one to construct a modification of $\Psi(z)$.

Slightly more generally, let $X$ be an infinite constructive world. Consider an optimal enumeration $u: \mathbf{Z}^{+} \rightarrow X$ in the sense of Kolmogorov or Schnorr (see 1.4.4 above). This means that $u$ is total recursive, surjective, and the function $C_{u}: X \rightarrow \mathbf{Z}^{+}$,

$$
C_{u}(x):=\min \{k \mid u(k)=x\}
$$

is (a representative of) Kolmogorov complexity of constructive objects of type $X$.

Now, define the Kolmogorov total order on $X$ associated to $u$ by

$$
x<y \Leftrightarrow C_{u}(x)<C_{u}(y)
$$

and denote by $\mathbf{K}=\mathbf{K}_{u}: X \rightarrow \mathbf{Z}^{+}$the function

$$
\mathbf{K}(x):=1+\operatorname{card}\left\{y \mid C_{u}(y)<C_{u}(x)\right\} .
$$


Clearly, $\mathbf{K}$ is a bijection. If we arrange $X$ in the order of growing Kolmogorov complexity, $\mathbf{K}(x)$ is precisely the number of $x$ in this order.

It is convenient also to introduce a Kolmogorov order on $\mathbf{Z}^{+}$. We will denote the respective numbering by the same letter $\mathbf{K}$. This should not lead to a confusion. have

It is straightforward to check that for some constant $c_{0}>0$ and all $x \in X$, we

$$
c_{0} C_{u}(x) \leq \mathbf{K}(x) \leq C_{u}(x) .
$$

Let now $\sigma: X \rightarrow X$ be a partial recursive map, such that $\sigma$ maps $D(\sigma)$ to $D(\sigma)$ and induces a permutation of this set. Put

$$
\sigma_{\mathbf{K}}:=\mathbf{K} \circ \sigma \circ \mathbf{K}^{-1}
$$

and consider this as a permutation of the subset

$$
D\left(\sigma_{\mathbf{K}}\right):=\mathbf{K}(D(\sigma)) \subset \mathbf{Z}^{+}
$$

consisting of numbers of elements of $D(\sigma)$ in the Kolmogorov order. We have then the following modified version of $(3.7)$ :

3.6.1. Proposition. Let $x \in D(\sigma)$. If the orbit $\sigma^{\mathbf{Z}}(x)$ is infinite, then there exist such constants $c_{1}, c_{2}>0$ that for $k:=\mathbf{K}(x)$ and all $n \in \mathbf{Z}$ we have

$$
c_{1} \cdot \mathbf{K}(n) \leq \sigma_{\mathbf{K}}^{n}(k) \leq c_{2} \cdot \mathbf{K}(n) .
$$

Proof. Let $k=\mathbf{K}(x), x \in X$. We have for $n>0$ :

$$
\sigma_{\mathbf{K}}^{n}(k)=\mathbf{K}\left(\sigma^{n}(x)\right) \leq c \cdot \mathbf{K}(n)
$$

for any fixed Kolmogorov complexity order on $\mathbf{Z}^{+}$(which we denote by the same letter $\mathbf{K}$ in order to simplify notation). In fact, if we replace $\mathbf{K}$ in (3.12) by the appropriate complexity $C$, this will follow from (2.1), since $n \mapsto \sigma^{n}(x)$ is an everywhere defined morphism $\mathbf{Z}^{+} \rightarrow X$ in $\mathcal{C}$. It remains to invoke (3.10).

Furthermore, let $Y:=\left\{\sigma^{n}(x) \mid n \in \mathbf{Z}^{+}\right\}$. This is a recursively enumerable subset of $X$, and the partial function $\lambda: X \rightarrow \mathbf{Z}^{+}$with definition domain $Y$

$$
\lambda(y)=n, \text { if } y=\sigma^{n}(x)
$$


is partial recursive. Hence again in view of (3.6) and (2.1),

$$
\mathbf{K}(n)=\mathbf{K}(\lambda(y)) \leq c^{\prime} \cdot \mathbf{K}(y)=c^{\prime} \cdot \mathbf{K}\left(\sigma^{n}(x)\right) .
$$

Combining (3.12) and (3.13), we get (3.11) for $n \geq 0$. Applying the same reasoning to $\sigma^{-1}$ in place of $\sigma$, we obtain (3.11) for negative $n$.

3.7. Proposition. With the same notations as in Proposition 3.6.1, put

$$
\Phi(k, \sigma ; z):=\frac{1}{k^{2}}+\sum_{n=1}^{\infty} \frac{z^{\mathbf{K}(n)}}{\left(\sigma_{\mathbf{K}}^{n}(k)\right)^{2}} .
$$

Then we have:

(i) If $\sigma^{\mathbf{Z}}$-orbit of $x$ is finite, then $\Phi(x, \sigma ; z)$ is a rational function in $z$ whose all poles are of the first order and lie at roots of unity.

(ii) If this orbit is infinite, then $\Phi(x, \sigma ; z)$ is the Taylor series of a function analytic at $|z|<1$ and continuous at the boundary $|z|=1$.

3.8. Remarks. (a) In the proofs of Propositions 3.6.1 and 3.5, we actually used only the fact that $\sigma$ restricted to the particular orbit $Y:=\left\{\sigma^{n}(x) \mid n \in \mathbf{Z}^{+}\right\}$ is recursive. thus justifying our choice of $\mathcal{A}_{-}$in 3.1 (b) above.

(b) Although Kolmogorov's order is as uncomputable as Kolmogorov's complexity, there are serious arguments for studying constructions, explicitly involving it, such as our renormalization characters.

One can argue that all cognitive activity of our civilization, based upon symbolic (in particular, mathematical) representations of reality, deals actually with the initial Kolmogorov segments of potentially infinite linguistic constructions, always replacing vast volumes of data by their compressed descriptions. This is especially visible in the outputs of the modern genome projects.

In this sense, such linguistic cognitive activity can be metaphorically compared to a gigantic precomputation process, shellsorting infinite worlds of expressions in their Kolmogorov order.

\section{References}

[BaSt] J. Baez, M. Stay. Physics, topology, logic and computation: a Rosetta stone. Preprint arXiv:0903.0340

[CalSt1] Ch. Calude, M. Stay. Most programs stop quickly or never halt. Adv. in Appl. Math., 40 (2008), 295-308 
[CalSt2] Ch. Calude, M. Stay. Natural halting probabilities, partial randomness, and zeta functions. Information and Computation, 204 (2006), 1718-1739.

[E-FMan] K. Ebrahimi-Fard and D. Manchon. The combinatorics of Bogolyubov's recursion in renormalization. math-ph/0710.3675

[GaLe] P. Gács, A. Levin. Causal Nets or What Is a Deterministic Computation? Int. Journ. Theor. Phys., vol. 21, No. 12 (1982), 961-971.

[Gr] J. Grass. Reasoning about Computational Resource Allocation. An introduction to anytime algorithms. Posted on the Crossroads website.

[GrZi] J. Grass, S. Zilberstein. Programming with anytime algorithms. In: Proc. of the IJCAI-95 Workshop on Anytime Algorithms and Deliberation Schedulyng. Montreal, 1995.

[He] A. Heller. An existence theorem for recursive categories. Journ. of Symb. Logic, vol. 55, No 3 (1990), 1252-1268.

[Le] L. Levin. Various measures of complexity for finite objects (axiomatic description). Soviet Math. Dokl., vol 17, No. 2 (1976), 522 -526.

[LiVi] Ming Li, P. Vitányi. An introduction to Kolmogorov complexity and its applications. Springer, 1993.

[Ma1] Yu. Manin. A Course in Mathematical Logic. Springer Verlag, 1977. XIII+286 pp. (The second, expanded Edition to be published in 2009).

[Ma2] Yu. Manin. Classical computing, quantum computing, and Shor's factoring algorithm. Séminaire Bourbaki, no. 862 (June 1999), Astérisque, vol 266, 2000, 375-404. quant-ph/9903008.

[Ma3] Yu. Manin. Renormalization and computation I. Motivation and background. Preprint arxiv:0904.492

[NaWe] A. Nabutovsky, S. Weinberger. The fractal nature of Riemm/Diff I. Geometriae Dedicata, 101 (2003), 145-250.

[Ro] H. Rogers. Gödel numberings of partial recursive functions. Journ. Symb. Logic, 23 (1958), 331-341.

[RuZi] S. J. Russell, S. Zilberstein. Composing real-time systems. In: Proc. of the XIIth International Joint Conference on Artificial Intelligence, (1991), Sydney, pp. 212-217.

[Sch] C. P. Schnorr. Optimal enumerations and optimal Gödel numberings. Math. Systems Theory, vol. 8, No. 2 (1974), 182-191.

[So] R. I. Soare. Computability theory and differential geometry. Bull. of Symb. Logic, vol. 10, Nr 4 (2004), 457-486. 
[Ya] N. S. Yanofsky. Towards a definition of an algorithm. math.LO/0602053 